Jörg Melzer ${ }^{\mathrm{a}, \mathrm{b}}$

Vor rund 20 Jahren begann mit der Akademisierung von Komplementärmedizin und Naturheilkunde an Universitäten in Europa ein entscheidender wissenschaftlicher Emanzipations- und Professionalisierungsprozess [1]. War vorher jahrhundertealtes Erfahrungswissen vor allem durch experimentelle und klinische Studien für die medizinische Alltagspraxis geprüft worden, ging es nun um die wissenschaftliche Integration von Komplementärmedizin und Naturheilkunde an Universitätskliniken und Lehrkrankenhäusern. Die Phytotherapie spielte dabei eine wichtige Rolle, da ihre Forschungen aufgrund der methodischen Nähe zur klinischen Pharmakologie besonders geeignet schienen, traditionsreiche Empirie durch klinische Studien zu analysieren [2, 3]. $\mathrm{Zu}$ diesem Prozess trug entschieden die Orientierung vieler komplementärmedizinisch Forschender an der Methodik der evidenzbasierten Medizin (EbM) bei [4]. Durch die in der Folge gewonnene Evidenz entwickelte sich die wissenschaftliche Akzeptanz für viele Interventionen aus traditionellen Medizinsystemen.

So stellt sich heute - auf der Grundlage einer mittlerweile breiten klinischen Evidenz, beispielsweise auf dem Gebiet der Phytotherapie - für unterschiedliche medizinische Fachbereiche (z.B. Innere Medizin, Onkologie, Psychiatrie) die Frage, welche Behandlungen in der klinischen Praxis geeignet sind und aufgenommen werden können $[5,6]$.

\footnotetext{
anstitut für Naturheilkunde,

${ }^{b}$ Klinik für Psychiatrie und Psychotherapie, UniversitätsSpital Zürich, Schweiz
}

\title{
Ergebnisse einer randomisierten, placebokontrollierten pharmako- klinischen Studie mit einem pflanzlichen Kombinationspräparat zur Behandlung von Patienten mit somatoformen Störungen
}

Pflanzliche Arzneimittel gehören zum Kulturgut der europäischen Komplementärmedizin und haben daher eine lange Praxisempirie. Während pflanzliche Extrakte als Monopräparate trotz ihres genuinen Vielstoffcharakters in präklinischen Untersuchungen oft gut untersucht sind und in klinischen Studien vielfach ihre Wirksamkeit und Verträglichkeit gezeigt haben, werden pflanzliche Kombinationspräparate wesentlich kritischer betrachtet. Dies mag mit der einige Jahrzehnte vorherrschenden pharmakologischen Sichtweise zusammenhängen, dass möglichst selektive Wirkmechanismen von einzelnen Wirksubstanzen eine besonders effektive physiologische Wirkung im menschlichen Organismus entfalten könnten. In der Psychiatrie hat dies beispielsweise dazu geführt, dass nach dem Bekanntwerden der Tatsache, dass ein Mangel an Neurotransmittern an der Entstehung der Depression beteiligt ist, für die Behandlung der Depression selektive Serotonin-Wiederaufnahmehemmer (SSRI, z.B. Citalopram) entwickelt wurden. Nach jahrelangen Praxiserfahrungen weiss man, dass die SSRI zwar wirksame Psychopharmaka sind und besser vertragen werden als die älteren trizyklischen Antidepressiva, aber keineswegs - wie erwartet - eine sehr hohe Wirksamkeit haben. Mittlerweile haben sich nicht nur Antidepressiva mit einem dualen Wiederaufnahmehemmer (Serotonin-Noradrenalin, SNRI,
z.B. Duloxetin), d.h. ein auf zwei Neurotransmittersysteme ausgerichteter Wirkmechanismus, bewährt. Daneben gibt es tetrazyklische Antidepressiva wie Mirtazapin, die über zwei Neurotransmitter und zusätzlich zwei weitere Rezeptorsysteme wirken. Angesichts dieser multiplen Wirkmechanismen verwundert die Diskussion über zukünftige synthetische Antidepressiva mit Wirkmechanismen an multiplen Zielorten (multi-target) nicht [7, 8]. Spätestens hier bietet sich eine Basis, um den möglichen Vorteil von Phytotherapeutika in die Diskussion einzubringen. Sie sind aufgrund des Vielstoffcharakters der pflanzlichen Extrakte per se als eine Art «dirty drug» anzusehen. Das heisst, jeder pflanzliche Extrakt ist genuin kein Monopräparat, sondern ein Kombinationspräparat mit vielen Inhaltsstoffen; dies wird in der Heilpflanzenkunde als Vielstoffgemisch bezeichnet. Vielstoffgemische setzen an mehreren biologischen Strukturen, d.h. Neurotransmittern und Rezeptorsystemen, an. Als eines der wichtigsten Beispiele sind hier Johanniskrautextrakte zu nennen Zubereitungen aus Johanniskraut gehören zu den am besten beforschten Arzneipflanzen. Aufgrund vielfältiger Forschung sind serotonerge, glutamaterge, noradrenerge oder dopaminerge Wirkmechanismen im zentralen Nervensystem untersucht. Ihre Wirksamkeit bei Patienten mit leichten bis mittelgradigen depressiven Episoden wurde bisher in über zwei
(๑) 2012 S. Karger GmbH, Freiburg
Dr. med. Jörg Melzer

Institut für Naturheilkunde

UniversitätsSpital Zürich

Rämistrasse 100, 8091 Zürich, Schweiz

Tel. +41 44 255-2460, Fax -4394

joerg.melzer@usz.ch 
Dutzend randomisierten, kontrollierten Studien und mehreren MetaAnalysen der Cochrane Collaboration [9-11] bestätigt. Gleichzeitig erwiesen sich die Zubereitungen aus Johanniskraut als besser verträglich als die SSRI. Somit ist die Hypothese erlaubt, dass Johanniskrautextrakte in niedrigeren Wirkstoffkonzentrationen durch mehrere, eventuell additive Effekte an verschiedenen Neurotransmitter- und Rezeptorsystemen des zentralen Nervensystems eine signifikante Gesamtwirksamkeit erzielen. Des Weiteren darf man spekulieren, dass die Wirkung an einzelnen Rezeptorsystemen (z.B. Serotonin- oder Noradrenalinrezeptoren) nicht vollständig ausgereizt wird und damit auch die Wahrscheinlichkeit von Nebenwirkungen reduziert ist, was die bessere Verträglichkeit erklären könnte. Johanniskrautextrakte als pflanzliche Vielstoffgemische sind somit «dirty drugs» mit rational erklärbarem Multi-target-Wirksamkeitsprofil. Das führte dazu, dass sie neben den bekannten SSRI in die Behandlungsleitlinien der deutschen und schweizerischen Fachgesellschaften für Psychiatrie und Psychotherapie zur Behandlung von Patienten mit leichten bis mittelgradigen unipolaren depressiven Episoden aufgenommen wurden [12-14].

Während dieses Beispiel zeigt, dass manche pflanzlichen Monopräparate mit Vielstoffcharakter gut beforscht und klinisch allgemein akzeptiert sind, werfen Kombinationspräparate trotz bewährter Praxisempirie vor dem Hintergrund der EbM die Frage nach Forschungsevidenz im Sinne des Wirksamkeitsnachweises auf.

Um einen klinischen Nachweis für das Rationale pflanzlicher Kombinationspräparate beizusteuern, planten wir eine klinische Studie [15], um exemplarisch an einem Arzneimittel vonseiten der klinischen Wirksamkeit einen Beitrag zur Kombinationsbegründung zu liefern.

Pflanzliche Zubereitungen aus den Extrakten von Melissenblättern, Passionsblume und Baldrianwurzel werden in Europa einzeln und teilweise in Kombination wegen ihrer beruhigenden und Angst reduzierenden Wirkung angewandt [16-21]. In der Schweiz ist die 4er-Kombination aus Extrakten von Baldrianwurzel, Melissenblättern, Passionsblume und Pestwurz zur Behandlung von Symptomen wie Nervosität, Spannungs- und Unruhezuständen, Prüfungsangst, krampfartigen $\mathrm{Ma}$ gen-Darm-Beschwerden, erhöhte Reizbarkeit sowie gelegentliche Ein- und Durchschlafstörungen zugelassen.

Die im Arzneimittel Relaxane (Ze 185) [22] enthaltenen einzelnen Pflanzendrogen bzw. die Extrakte aus ihnen sind allesamt gut untersucht. Für Pestwurz (Petasites rhizoma) werden Effekte der Oxopetasanester Petasin und Isopetasin auf die Hemmung der Biosynthese von Leukotrinen im Sinne möglicher spasmolytisch-entspannender Wirkung diskutiert [23-25].

Mit Zubereitungen aus Melissenblättern (Melissae folium) zeigten sich Wirkungen auf zentralnervöse kognitive Prozesse, die über Effekte am cholinergen System (nikotinerge, muskarinerge Rezeptoren) oder GABA(Gamma-Aminobuttersäure)System erklärt werden können.

Für Extrakte aus der Passionsblume (Passiflorae herba) fand man eine anxiolytische Wirksamkeit, wobei sedativ-anxiolytische Effekte über das Flavonoid Chrysin oder GABARezeptorwirkungen diskutiert werden, aber noch nicht abschliessend geklärt sind.

Von Baldrian (Valerianae radix) sind beruhigende Wirkungen bei Unruhezuständen und nervösen Einschlafstörungen bekannt und werden unter anderem über zentralnervöse Effekte auf Adenosin- und GABA-Rezeptoren beschrieben.

Im pflanzlichen Arzneimittel Ze 185 sind nun diese 4 Pflanzenextrakte, mit experimentell gut untersuchten physiologischen Effekten an unterschiedlichen Rezeptorsystemen, kombiniert. Das bedeutet, dass ein komplexes Vielstoffgemisch vorliegt.
Interessanterweise liegen die jeweiligen Dosierungen der 4 einzelnen Pflanzendrogen im Kombinationspräparat niedriger als die empfohlene Dosierung für den jeweiligen $\mathrm{Ge}$ brauch als Monopräparat. Aufgrund dieses Dosierungsunterschieds stellt sich die Frage, ob durch die Kombination von 4 Pflanzenextrakten in niedriger Dosis quasi eine eigene Wirkstoffkombination entsteht. Anstatt eines präklinischen experimentellen Modells wählten wir nun eine klinische Studie, um dieser Frage nachzugehen.

In einer randomisierten, placebokontrollierten, doppelblinden Studie [15] mit 182 Patienten, die an somatoformen Störungen litten, wurde ein dreiarmiges Studiendesign gewählt, um exemplarisch die Wirksamkeit der bekannten 4er-Kombination im Vergleich zur 3erKombination ohne Pestwurz und zur Placebobehandlung zu untersuchen. Als primäre Zielparameter dienten, wie bei somatoformen Störungen (vor allem undifferenzierte Somatisierungsstörungen - F45.1, aber auch Somatisierungsstörungen - F45.0) in klinischen Studien verwendet, die Reduktion der Symptome Angst (visuelle Analogskala, VAS) und Depression (Beck's Depression Inventory, BDI) [26]. Als sekundäre Zielparameter wurden Clinical Global Impression (CGI) sowie Arzt- und Patienteneinschätzung untersucht. Es zeigte sich in der Kurzzeitintervention (14 Tage) bei einer Tagesdosis von 3 Tabletten, dass sowohl die 4er-Kombination signifikant wirksamer war als die 3er-Kombination ohne Pestwurz als auch beide Kombinationspräparate wirksamer waren als Placebo ( $\mathrm{p}=0,001$ bezüglich Symptomreduktion sowohl für Depression als auch Angst im Vergleich von Ze 185 vs. Placebo). Somit konnte der signifikante zusätzliche Nutzen des Pestwurzextrakts in der 4er-Kombination gegenüber der 3er-Kombination ohne Pestwurz gezeigt werden (4er-Kombination $>$ 3er-Kombination $>$ Placebo). Daher darf angesichts 
der klinischen Forschungsevidenz spekuliert werden, ob der Gesamtextrakt der 4er-Kombination somit als eigene Wirkstoffkombination angesehen werden kann.

Bezüglich Verträglichkeit und Sicherheit traten keine schwerwiegenden unerwünschten Ereignisse auf. Die nicht schwerwiegenden unerwünschten Ereignisse unterschieden sich nicht signifikant zwischen den Behandlungsgruppen und betrafen bei jenen mit möglichem Zusammenhang zur Intervention hauptsächlich den Gastrointestinaltrakt (z.B. zweimal Übelkeit und je einmal Erbrechen, Blähungen, Verstopfung).

Das pflanzliche Kombinationspräparat bewirkt demnach in der Kurzzeitanwendung, etwa zur Überbrückung bis zum Beginn einer Psychotherapie (Therapie der ersten Wahl), eine signifikante und auch klinisch relevante Reduktion der untersuchten Symptomparameter Angst und Depression bei Patienten mit somatoformen Störungen.

Klinische Studien zu pflanzlichen Kombinationspräparaten können, wie gezeigt, nicht nur zur Evidenz von Wirksamkeit und Verträglichkeit, sondern partiell auch zur Rationale für die Kombinationsbegründung beitragen. Nun könnte man fordern, jeweils ähnliche klinische Studien etwa ohne die Extrakte von Passionsblume, Melissenblättern und Baldrianwurz durchzuführen, um die Kombination bis ins Detail zu begründen. Dies würde aber, angesichts der gezeigten klinischen Wirksamkeit im Vergleich zu einer Placebobehandlung, eher ein Forschungsinteresse stillen, als dass es einen zusätzlichen klinischen Nutzen für die Patienten hätte.

In der Praxis zeigt sich, dass auch Patienten mit psychischen Störungen komplementärmedizinische Interventionen zur Linderung der Beschwerden verwenden. Nach Umfragen sind es bis zu 30\% der Patienten mit depressiven und $49 \%$ mit ängstlichen
Symptomen, die Komplementärmedizin zumindest supportiv neben anderen Behandlungsansätzen nutzen [27, 28]. Daher müssen entsprechende komplementärmedizinische Interventionen, auch pflanzliche Kombinationspräparate, evaluiert werden, um eine Behandlungs- und Arzneimittelsicherheit für Patienten und Ärzte zu gewährleisten.

\section{Literatur}

1 Melzer J, Brignoli R, Saller R: Komplementärmedizin, Phytotherapie und Sojaisoflavone als Phytoöstrogene. Zentralbl Gynakol 2004;126:138-147.

-2 García-García P, López-Muñoz F, Rubio G, Martín-Agueda B, Alamo C: Phytotherapy and psychiatry: bibliometric study of the scientific literature from the last 20 years. Phytomedicine 2008;15:566-576.

3 Melzer J, Saller R: Gibt es ein bestimmtes Menschenbild in der Naturheilkunde / Komplementärmedizin? Forsch Komplementmed 2006;13:210-219.

4 Sackett D, Strauss S, Richardson W, Rosenberg W, Haynes R: Evidence-Based Medicine. How to Practice and Teach EBM, ed 2. Edinburgh, Churchill Livingstone, 2000.

5 Kenny E, Muskin PR, Brown R, Gerbarg PL: What the general psychiatrist should know about herbal medicine. Curr Psychiatry Rep 2001;3:226-234

6 Werneke U, Turner T, Priebe S: Complementary medicines in psychiatry: review of effectiveness and safety. Br J Psychiatry 2006;188:109-121.

7 Millan MC: Multi-target strategies for the improved treatment of depressive states: conceptual foundations and neuronal substrates, drug discovery and therapeutic application. Pharmacol Ther 2006;110:135370 .

8 Wong EH, Nikam SS, Shahid M: Multiand single-target agents for major psychiatric diseases: therapeutic opportunities and challenges. Curr Opin Investig Drugs 2008;9:28-36.

9 Linde K, Ramirez G, Mulrow CD, Pauls A, Weidenhammer W, Melchart D: St John's wort for depression - an overview and meta-analysis of randomised clinical trials. BMJ 1996;313:253-258.

10 Linde K, Mulrow CD, Berner M, Egger M: St John's wort for depression. Cochrane Database Syst Rev 2005;2:CD000448.

11 Linde K, Berner MM, Kriston L: St John's wort for major depression. Cochrane Database Syst Rev 2008;4:CD000448.

12 Harter M, Klesse C, Bermejo I, et al.: Evidenzbasierte Therapie der Depression. Die S3-Leitlinie unipolare Depression. Nervenarzt 2010;81:1049-1068.
13 Holsboer-Trachsler E, Hättenschwiler J, Beck J, Brand S, Hemmeter UM, Keck ME, Rennhard S, Hatzinger M, Merlo M, Bondolfi G, Preisig M, Seifritz E, Attinger Andreoli Y, Gehret A, Bielinski D: Die somatische Behandlung der unipolaren depressiven Störungen. Schweiz Med Forum 2010;10:802-809.

14 Melzer J, Keck ME: Phytotherapie in der Psychiatrie - gestern, heute, morgen. Ars medici Thema Phytotherapie 2011;1:16-20.

15 Melzer J, Schrader E, Brattström A, Schellenberg R, Saller R: Fixed herbal drug Combination with and without butterbur (Ze 185) for the treatment of patients with somatoform disorders: randomized, placebo-controlled pharmacoclinical trial. Phytother Res 2009;23:1303-1308.

16 Carlini EA: Plants and the central nervous system. Pharmacol Biochem Behav 2003; 75:501-512.

17 European Scientific Cooperative On Phytotherapy (ESCOP) (ed): Melissae folium (Melissa Leaf). Stuttgart, Thieme, 2003, pp 324-328.

18 ESCOP: Passiflorae herba (Passion Flower). Stuttgart, Thieme, 2003, pp 359-364.

19 ESCOP: Valerianae radix (Valerian Root). Stuttgart, Thieme, 2003, pp 539-546.

20 Dhawan K, Dhawan S, Sharma A: Passiflora: a review update. J Ethnopharmacol 2004;94:1-23.

21 Kennedy DO, Little W, Scholey AB: Attenuation of laboratory-induced stress in humans after acute administration of $\mathrm{Me}$ lissa officinalis (lemon balm). Psychosom Med 2004;66:607-613.

22 Arzneimittel-Kompendium der Schweiz ${ }^{\circledR}$. Relaxane. Basel, Documed AG 2011. www.kompendium.ch/MonographieTxt. aspx?lang $=$ de\&MonType $=f$.

23 Bickel D, Roder T, Bestmann HJ, Brune $\mathrm{K}$ : Identification and characterization of inhibitors of peptido-leukotriene-synthesis from Petasites hybridus. Planta Med 1994;60:318-322.

24 Kälin P: Gemeine Pestwurz (Petasites hybridus) - Portrait einer Arzneipflanze. Forsch Komplementärmed Klass Naturheilkd 2003; 10(suppl 1):41-44.

25 Giles M, Ulbricht, Khalsa KP, Kirkwood CD, Park, Basch E: Butterbur: an evidence-based systematic review by the natural standard research collaboration. J Herb Pharmacother 2005;5:119-143.

26 Dilling $\mathrm{H}$, Mombour $\mathrm{W}$, Schmidt $\mathrm{MH}$ (Hrsg): Weltgesundheitsorganisation. Internationale Klassifikation psychischer Störungen. ICD-10 Kapitel V (F). Klinischdiagnostische Leitlinien, ed 5. Bern, Hans Huber, 2005.

-27 Unützer J, Klap R, Sturm R, Young AS, Marmon T, Shatkin J, Wells KB: Mental disorders and the use of alternative medicine: results from a national survey. Am J Psychiatry 2000;157:1851-1857.

28 Wahlström M, Sihvo S, Haukkala A, Kiviruusu O, Pirkola S, Isometsa E: Use of mental health services and complementary and alternative medicine in persons with common mental disorders. Acta Psychiatr Scand 2008;118:73-80. 